\title{
A case of broad ligament leiomyoma presenting as an ovarian mass
}

\author{
Rajesh Kumari*, Vandana, Vidushi Kulshrestha, Jai Bhagwan Sharma, Alka Kriplani
}

Department of Obstetrics and Gynecology, All India Institute of Medical Sciences, New Delhi, India

Received: 16 March 2017

Accepted: 21 April 2017

\author{
*Correspondence: \\ Dr. Rajesh Kumari, \\ E-mail: drrajeshkumari@yahoo.com
}

Copyright: (c) the author(s), publisher and licensee Medip Academy. This is an open-access article distributed under the terms of the Creative Commons Attribution Non-Commercial License, which permits unrestricted non-commercial use, distribution, and reproduction in any medium, provided the original work is properly cited.

\begin{abstract}
The broad ligament is the commonest extra uterine site for the occurrence of leiomyoma but with a very low incidence rate. It poses both clinical and radiological challenge in differentiating from an ovarian tumour. A 25-yearold unmarried female presented with history of lower abdominal pain associated with rapidly increasing abdominal distension for last 3 months. General physical examination was unremarkable. Examination of the abdomen revealed a firm, non-tender mass, with limited mobility arising from the pelvis corresponding to a uterine size of 32 weeks. Ultrasonography of abdomen revealed a $20 \times 17 \times 11 \mathrm{~cm}$ right adnexal multi-loculated cyst. Contrast enhanced computer tomography scan of abdomen and pelvis showed a $12 \times 17 \times 17 \mathrm{~cm}$ well defined cystic lesion arising from the pelvis and ascending in to the abdominal cavity. The lesion also showed internal septa and peripheral rim enhancement. Right ovary was not seen separately. Tumor markers including CA-125 (22.4 IU/ ml), CEA (1.83/ml), CA-19.9 $(22 \mathrm{U} / \mathrm{ml})$, Beta HCG $(1.20 \mathrm{IU} / \mathrm{ml})$, LDH $(1.17 \mathrm{IU} / \mathrm{ml})$, and alpha feto-protein $(0.8 \mathrm{ng} / \mathrm{ml})$ were within normal limit. Laparotomy revealed a cystic mass arising from the right broad ligament. Histopathological examination revealed a broad ligament leiomyoma with extensive cystic degeneration. Broad ligament leiomyoma is uncommon tumour of pelvis and its differentiation from ovarian masses may be challenging for the clinicians.
\end{abstract}

Keywords: Broad ligament, Leiomyoma, Ovarian tumour, Pelvic tumour

\section{INTRODUCTION}

Leiomyomas are the most common benign tumors of female pelvis during reproductive age affecting approximately $20-30 \%$ of woman aged 30 years or more. ${ }^{1}$ These tumors can vary in size from microscopic to size of full term gravid uterus. The primary site of origin of pelvic leiomyomas is usually uterus, however these can arise from extra uterine sites in pelvis as well as in the abdomen. $^{2}$

Extra uterine leiomyomas although rare, however present a greater diagnostic challenge. Broad ligament is the commonest extra uterine site where leiomyomas are found, but its overall incidence is rare. ${ }^{2}$ One of the known feature of leiomyomas is that as these tumors enlarge and outgrow their blood supply, these undergo various types of degeneration named hyaline, myxoid, calcific, cystic, or red degeneration. ${ }^{1}$ When cystic degeneration is present in broad ligament leiomyoma, it may be confused with cystic adnexal tumor and may lead to problem in further management. Here we present a case of giant broad ligament leiomyoma with extensive cystic degeneration mimicking a pelvic adnexal tumor.

\section{CASE REPORT}

A 25-year-old unmarried female presented with history of lower abdominal pain associated with rapidly increasing abdominal distension for last 3 months. She had regular menstrual cycle and normal bowel and bladder habit. She also denied any history of anorexia or weight loss. She had no history of previous medical or surgical illness. There was no history of malignancy in the family. General physical examination was unremarkable. Examination of the abdomen revealed a firm, non-tender 
mass with limited mobility arising from the pelvis corresponding to a uterine size of 32 weeks. Ultrasonography (USG) of abdomen revealed a $20 \times 17 \times 11 \mathrm{~cm}$ right adnexal multi-loculated cyst. The differentials diagnoses suggested on USG findings included mucinous cyst adenoma or cyst adenocarcinoma of ovary. Contrast enhanced computer tomography (CECT) scan of abdomen and pelvis was done for further evaluation. It showed a $12 \times 17 \times 17 \mathrm{~cm}$ well defined cystic lesion arising from the pelvis and ascending in the abdominal cavity (Figure 1).

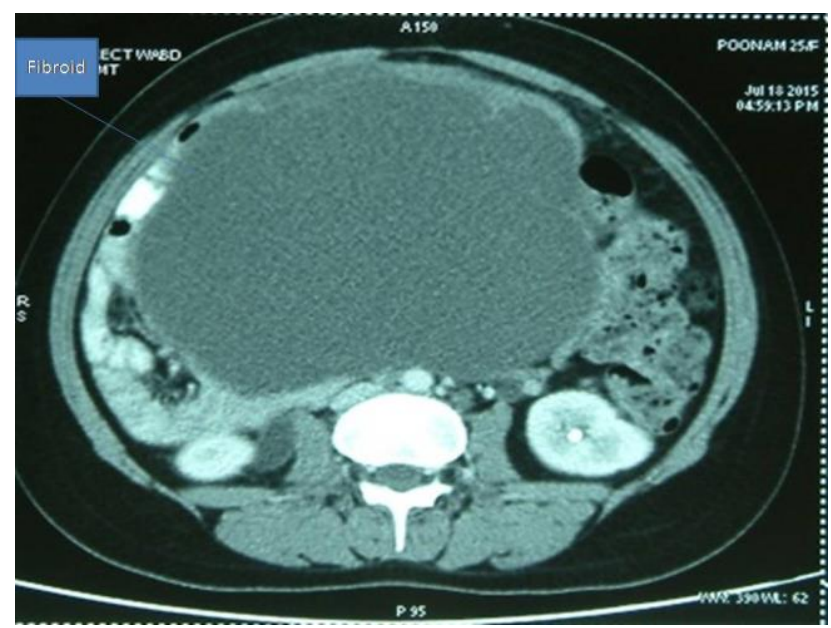

Figure 1: Contrast enhanced computer tomography (CECT) scan of abdomen and pelvis showing a $12 \times 17 \times 17 \mathrm{~cm}$ well defined cystic lesion arising from the pelvis and ascending in the abdominal cavity.

The lesion also showed internal septa and peripheral rim enhancement. Right ovary was not seen separately. The uterus was normal in size and grossly deviated to the left. The left ovary was normal. There was no free fluid seen in the abdomen or pelvis. Also, there were no features suggestive of regional or distant nodal metastasis. Routine laboratory testing including complete blood count, liver function test and renal function test and radiograph of the chest were normal. Tumor markers including CA-125 (22.4 IU/ ml), CEA (1.83/ml), CA$19.9(22 \mathrm{U} / \mathrm{ml})$, Beta HCG (1.20IU/ $\mathrm{ml})$, LDH $(1.17 \mathrm{IU} / \mathrm{ml})$, and alpha feto-protein $(0.8 \mathrm{ng} / \mathrm{ml})$ were within normal limit. On the basis of clinical examination and radiological findings a provisional diagnosis of primary ovarian tumor was made and patient underwent laparotomy. Laparotomy was done by infra umbilicus midline vertical incision. Laparotomy revealed a cystic mass arising from the right broad ligament. The uterus, bilateral tubes and ovaries were normal (Figure 2). One litre of sero-mucinous fluid was drained from the mass before removal. Enucleation of cyst was performed via an incision in to the anterior leaf of broad ligament. Gross pathologic examination revealed a $16 \times 12 \mathrm{~cm}$ globular cystic structure in broad ligament with multiple fluid filled pocket. Histopathological examination revealed a broad ligament leiomyoma with extensive cystic degeneration.

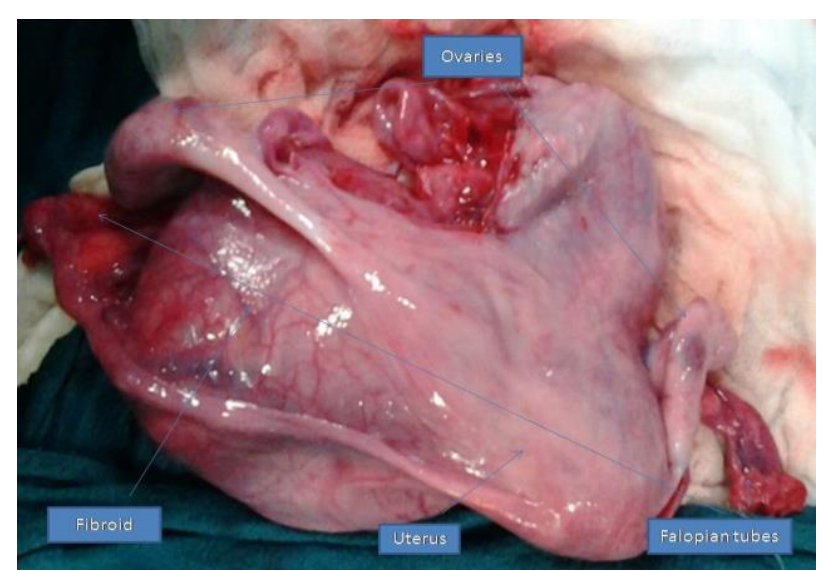

Figure 2: Laparotomy showing a cystic mass arising from the right broad ligament with normal uterus, bilateral tubes and ovaries.

\section{DISCUSSION}

Extra uterine leiomyomas as such are very uncommon, although, broad ligament is the commonest site for extra uterine leiomyomas. These benign smooth muscle tumors from broad ligament are usually hormone sensitive. Being benign in nature, these tumors are usually asymptomatic. However, if the leiomyoma reaches significant size, it produces pressure symptoms. It can push uterus to contralateral side or can potentially compress the surrounding pelvis structures and manifest clinically with various sign and symptoms. ${ }^{4,5}$ Our patient presented with abdomen pain likely due to huge mass and heaviness in the lower abdomen. However, there were no signs suggestive of compression of other organs.

Management of patients with a large pelvis mass include a work-up for differential diagnoses. Differential diagnoses of broad ligament leiomyoma include benign and malignant tumours of ovarian origin, tubo-ovarian masses, broad ligament cyst, peritoneal inclusion cyst, haematoma, abscess, lymphocele and lymphadenopathy. ${ }^{1,2}$ USG is very useful investigational tool which can help in finding the origin of mass and narrowing down the differential diagnosis. However, when mass is large it may be challenging to delineate the exact origin of the mass and leads to problem in diagnosis. $^{5-7}$ The USG findings of the index case suggested that the mass was of ovarian origin which was indeed a broad ligament tumour. CT scan and magnetic resonance imaging may provide further details and may be useful in most of the cases. Leiomyoma can undergo secondary changes which include degeneration, infarction, necrosis, haemorrhage and rarely sarcomatous changes. Among these cystic degeneration, as present in this case, is said to be found in $4 \%$ of all. ${ }^{1,6}$ These changes may also alter the radiological features and lead to confusion in diagnosis. In this case, radiological appearance on USG and CT scan of abdomen were suggestive of extensive cystic tumour arising from right adenexa with normal uterus and left ovary raise the 
suspicion of right ovarian neoplasm. This case highlights that in many cases the exact diagnosis is only made during laparotomy. ${ }^{5,7,8}$ Once diagnosed, leiomyomas have very good prognosis. Surgery may be challenging in few cases due to enormous size. The attention is required to avoid the injury to surrounding structures such as ureter, bladder and intestine.

\section{CONCLUSION}

Broad ligament leiomyomas are uncommon pelvis tumours. Broad ligament leiomyoma, especially when very large in size, often mimic malignant tumours at imaging. Most commonly it mimics ovarian tumors. Our case highlights that broad ligament leiomyoma should be kept as differential diagnosis for adnexal or ovarian mass.

Funding: No funding sources Conflict of interest: None declared

Ethical approval: Not required

\section{REFERENCES}

1. Dutta DC, Konar H. Benign lesion of uterus In: DC Dutta's Textbook of Gynecology. $7^{\text {th }}$ ed. India: Jaypee Brothers; 2016:272-5.
2. Kumar P, Malhotra N. Tumours of the corpus uteri and tumours of the pelvic ligament. In: Jeffcoat's Principles of Gynaecology 7th ed. New Delhi: Jaypee Brothers Medical Publishers (P) Ltd.; 2008:487-516.

3. Cramer SF, Patel A. The frequency of uterine leiomyomas. Am J Clini Pathol. 1990;94(4):435-8.

4. Stewart EA. Uterine Fibroids. Lancet. 2001;357(9252);293-8.

5. Rajput DA, Gedam JK. Broad Ligament Fibroid: A Case Series. IJSS. 2015;1(11):8-11.

6. Asotra S, Kaushik R, Gulati A. Broad ligament fibroid with neurilemoma like pattern. J K Sci. 2009:11-215.

7. Godbole RR, Lakshmi KS, Vasant K. Rare case of giant broad ligament fibroid with myxoid degeneration. J Sci Soc. 2012;39(3):144-46.

8. Low SC, Chong CL. A case of cystic leiomyoma mimicking an ovarian malignancy. Ann Med Singapore. 2004;33;371-4.

Cite this article as: Kumari $\mathrm{R}$, Vandana, Kulshrestha V, Sharma JB, Kriplani A. A case of broad ligament leiomyoma presenting as an ovarian mass. Int J Reprod Contracept Obstet Gynecol 2017;6:2635-7. 